\title{
Evaluation of Prognosis of Brain Function with Early Transcranial Color Doppler Ultrasound in Patients after Cardiopulmonary Resuscitation
}

\author{
Hui Guo, Zhangshun Shen, Ning Xu, Qian Zhao, Hongling Li, Yangjuan Jia, Jianguo Li* $\mathbb{C}$ \\ Department of Emergency Medicine, Hebei General Hospital, Shijiazhuang, China \\ Email: *lijg65@163.com
}

How to cite this paper: Guo, H., Shen, Z.S., Xu, N., Zhao, Q., Li, H.L., Jia, Y.J. and Li, J.G. (2020) Evaluation of Prognosis of Brain Function with Early Transcranial Color Doppler Ultrasound in Patients after Cardiopulmonary Resuscitation. World Journal of Cardiovascular Diseases, 10, 658-665. https://doi.org/10.4236/wjcd.2020.109063

Received: September 7, 2020

Accepted: September 21, 2020

Published: September 24, 2020

Copyright () 2020 by author(s) and Scientific Research Publishing Inc. This work is licensed under the Creative Commons Attribution International License (CC BY 4.0).

http://creativecommons.org/licenses/by/4.0/

\section{(c) (i) Open Access}

\begin{abstract}
Objective: To evaluate the clinical value of transcranial color Doppler ultrasound (TCCD) in assessing cerebral function after cardiopulmonary resuscitation (CPR). Methods: A prospective study was conducted in 52 patients with cardiac arrest treated by CPR from January 2018 to January 2020, and its clinical data were analyzed. According to classification of cerebral performance category (CPC), 31 cases (CPC grade $1-2$ ) were selected in the good prognosis group and 21 cases (CPC grade $3-5)$ in the poor prognosis group. The cerebral blood flow was measured by transcranial Doppler ultrasound (TCCD) $24 \mathrm{~h}$ after CPR, and the differences were compared between the two groups in stroke index, diastolic blood flow velocity (Vd), systolic peak blood flow velocity $(\mathrm{Vs})$ and mean peak blood flow velocity $(\mathrm{Vm})$. The ROC curve of cerebral blood flow after CPR was drawn to predict the prognosis of brain function. Results: The data showed that the pulsatility index of middle cerebral artery of the poor prognosis group decreased within $24 \mathrm{~h}$; the difference between the two groups was statistically significant $(\mathrm{p}<0.05)$; the Vd, Vs, Vm increased in the good prognosis group; the difference between the two groups was statistically significant $(\mathrm{p}<0.05)$. The ROC curve of cerebral blood flow after CPR was drawn to predict the prognosis of brain function, and the results showed that the area under the curve and the optimal critical value of cerebral blood flow were 0.731 and 5.69 . The sensitivity and specificity were $67.3 \%$ and $79.1 \%$ respectively. Conclusion: The cerebral blood flow increase in the early stage of successful CPR is positively correlated with the prognosis of cerebral functional resuscitation. Monitoring intracranial blood flow after CPR by TCCD has clinical value to evaluate prognosis of brain function.
\end{abstract}

\section{Keywords}

Cardiopulmonary Resuscitation (CPR), Transcranial Color Bifunctional Ultrasound (TCCD), Cerebral Blood Flow, Prognosis of Brain Function 


\section{Introduction}

Cardiopulmonary resuscitation $(\mathrm{CPR})$ is the only effective treatment for cardiac arrest. With the development of CPR technology in China, the success rate of CPR has increased. Nevertheless, in practice, it was found that $45 \%-70 \%$ of the surviving patients after CPR developed ischemic hypoxic encephalopathy, showing severe neurological impairment and even brain death [1]. Therefore, early evaluation of brain function has important reference value for patients' prognosis [2]. The early establishment and maintenance of cerebral circulation at a certain level after CPR plays an important role in the recovery of cerebral function, and whether it is cerebral ischemia or cerebral congestion can cause secondary brain injury [3]. So changes in cerebral circulation and intracranial pressure are required dynamic assessment and real-time monitoring. Transcranial Doppler ultrasound (TCCD) is a convenient, rapid and noninvasive way to monitor changes of the spectrum and waveform morphology of the blood flow in middle cerebral artery [4]. It provides a reference for clinical indirect evaluation of intracranial pressure and cerebral perfusion [5]. From January 2018 to January 2020, 52 patients with cardiac arrest who were successfully rescued by CPR were selected as subjects and their clinical data were analyzed. The results are summarized as follows.

\section{Information and Methods}

\subsection{General Data}

First, confirm that you have the correct template for your paper size. This template has been tailored for output on the custom paper size $(21 \mathrm{~cm} * 28.5 \mathrm{~cm})$. This prospective observational study was performed in the emergency department of the Hebei General hospital from January 2018 and January 2020. Fifty-two patients suffering from cardiac arrest treated by CPR were included. They were successfully resuscitated from cardiac arrest and their clinical data were analyzed retrospectively. But return of spontaneous circulation (ROSC) does not automatically restore cerebral perfusion. This renders the brain at risk for ischemia and secondary brain injury. There were 35 males $(67.31 \%)$ and $17 \mathrm{fe}$ males $(32.69 \%)$, aged from 21 to $79(48.86 \pm 20.3)$ years old. Etiology: cardiovascular diseases in 18 cases (34.62\%), acute poisoning in 12 cases $(23.08 \%)$, respiratory diseases in 11 cases $(21.15 \%)$, cerebrovascular diseases in 7 cases (13.46\%), and other diseases in 4 cases (7.69\%). Inclusion criteria: Patients who recovered spontaneous circulation after CPR in our department. 1) Age $\geq 18$ years old; 2) At least one intact eyeball; 3) No ophthalmic, cervical vascular and craniocerebral surgery in the last 12 months. Exclusion criteria [2] [3]: 1) Age > 80 years old or $<18$ years old; 2) Deaths within $24 \mathrm{~h} ; 3$ ) Patients with craniocerebral trauma; 4) Maternity; 5) Dissatisfied transcranial Doppler ultrasound (TCD) spectrum; Referring to cerebral performance category scoring (CPC), CPC1 - 2 showed good prognosis of nerve function and CPC 3 - 5 showed poor prognosis of nerve function (Table 1) [6]. The 52 cases were divided into 31 
Table 1. Cerebral performance category (CPC) score scale [1].

\begin{tabular}{|c|c|c|}
\hline Grading & Neurofunctional manifestations & Clinical manifestations \\
\hline CPC 1 & Good cerebral performance: & $\begin{array}{l}\text { Conscious, alert, able to work, might have } \\
\text { mild neurologic or psychologic deficit. }\end{array}$ \\
\hline СРC 2 & Moderate cerebral disability & $\begin{array}{l}\text { Conscious, sufficient cerebral function for } \\
\text { independent activities of daily life. } \\
\text { Able to work in sheltered environment. }\end{array}$ \\
\hline CPC 3 & Severe cerebral disability & $\begin{array}{l}\text { Conscious, dependent on others for daily support } \\
\text { because of impaired brain function. Ranges from } \\
\text { ambulatory state to severe demaletia or paralysis. }\end{array}$ \\
\hline CPC 4 & Coma or vegetative state & $\begin{array}{l}\text { Any degree of coma without the presence of } \\
\text { all brain death criteria. Unawareness, even if } \\
\text { appears awake (vegetative state) without } \\
\text { interaction with environment; may have } \\
\text { spontaneous eye opening and } \\
\text { sleep/awake cycles. Cerebral unresponsiveness. }\end{array}$ \\
\hline CPC 5 & Brain death & Apnea, areflexia, EEG silence, etc. \\
\hline
\end{tabular}

cases (the good prognosis group, CPC 1 - 2) and 21 cases (the poor prognosis group, CPC 3 - 5). This study was approved by the hospital ethics committee. All cases in this study were examined and treated with informed consent signed by patients and their families.

\subsection{Methods}

The template is used to format your paper and style the text. All margins, column widths, line spaces, and text fonts are prescribed; please do not alter them. You may note peculiarities. For example, the head margin in this template measures proportionately more than is customary. This measurement and others are deliberate, using specifications that anticipate your paper as one part of the entire journals, and not as an independent document. Please do not revise any of the current designations.

TCCD monitoring: Patients in supine position, who were placed $4 \mathrm{MHz}$ Doppler probes from the external orbital margin and temporal window between ears within $24 \mathrm{~h}$ after successful CPR. The temporal window between the outer edge of the orbit, the ear and the left zygomatic arch was taken as the observation point to obtain the best middle cerebral artery blood flow signal. Record the PI value and refer to formula 10/1.47 PI and calculate cerebral blood flow [3]. Measure and record middle cerebral artery diastolic blood flow velocity (Vd), systolic peak blood flow velocity (Vs), mean peak blood flow velocity (Vm), pulsatility index (PI).

\subsection{Observation Indicators}

The Vd, Vs, Vm, PI of middle cerebral artery was recorded within $24 \mathrm{~h}$ after successful CPR in the good prognosis group and the poor prognosis group. Draw the ROC curve of cerebral blood flow after CPR to predict the prognosis of 
brain function.

\subsection{Statistical Analysis}

All data were analyzed by SPSS 20.0 statistical software. The measured data were represented by mean standard deviation ( $\bar{x} \pm \mathrm{s}$ ), independent sample $T$ test was used for the comparison between the two groups of data with normal distribution and homogeneity of variance, and non-parametric test was used for the comparison between two groups that did not conform to normal distribution. Pearson correlation analysis was used for normal distribution data and $\chi^{2}$ test was used for counting data. $\mathrm{P}<0.05$ was statistically significant.

\section{Results}

In the good prognosis group, 22 male, 9 female, the mean age $(46.4 \pm 21.81)$ years; Etiology: Cardiovascular disease in 10 cases, Acute poisoning in 8 cases, Respiratory diseases in 7 cases; Cerebrovascular disease in 4 cases, other diseases in 2 cases. In the poor prognosis group, 13 male, 8 female, the mean age (47.8 \pm 18.92) years; Etiology: Cardiovascular disease in 8 case, Acute poisoning in 4 cases, Respiratory diseases in 4 cases, Cerebrovascular disease in 3 cases, other diseases in 2 cases. General data between groups were not statistically significant $(\mathrm{p}>0.05)$ (Table 2).

The parameter of cerebral blood flow within $24 \mathrm{~h}$ showed that the diastolic blood flow velocity $(\mathrm{Vd})$, systolic peak blood flow velocity (Vs) and mean peak blood flow velocity (Vm) significantly increased in the good prognosis group, and were statistically significant compared with the poor prognosis group ( $\mathrm{p}<$ 0.05) (Table 3).

Table 2. General information on patients in two groups.

\begin{tabular}{|c|c|c|c|}
\hline & $\begin{array}{l}\text { the good } \\
\text { prognosis } \\
\text { group n }(\%)\end{array}$ & $\begin{array}{l}\text { the poor } \\
\text { prognosis } \\
\text { group n (\%) }\end{array}$ & $P$ value \\
\hline Cases $(n=52)$ & $31(59.6 \%)$ & $21(40.4 \%)$ & \\
\hline Age (years) & $46.4 \pm 21.81$ & $47.8 \pm 18.92$ & 0.119 \\
\hline Gender (male/female) & $22 / 9$ & $13 / 8$ & 0.515 \\
\hline Cardiovascular and Cerebrovascular Diseases $(\mathrm{n}=18)$ & $10(19.23 \%)$ & $8(15.38 \%)$ & 0.302 \\
\hline Acute poisoning $(\mathrm{n}=12)$ & $8(15.38 \%)$ & $4(7.69 \%)$ & 0.551 \\
\hline Diseases of the respiratory system $(\mathrm{n}=11)$ & $7(13.46 \%)$ & $4(7.69 \%)$ & \\
\hline Cerebrovascular disease $(n=7)$ & $4(7.69 \%)$ & $3(5.76 \%)$ & 0.452 \\
\hline Other diseases $(n=4)$ & $2(3.84 \%)$ & $2(3.84 \%)$ & \\
\hline Initial monitoring of body temperature $\left(\begin{array}{ll}\bar{x} & \pm s\end{array}\right)$ & $36.81 \pm 1.41$ & $37.01 \pm 1.55$ & 0.154 \\
\hline $\mathrm{GCS}^{*}$ score ICU entry $(\bar{x} \pm s)$ & $4.27 \pm 1.24$ & $3.21 \pm 1.14$ & 0.125 \\
\hline CPC score $\left(\begin{array}{ll}\bar{x} & \pm s\end{array}\right)$ & $1.67 \pm 0.82$ & $4.70 \pm 0.48$ & 0.001 \\
\hline
\end{tabular}

*GCS: Glasgow Coma Scale. 
Compared with the poor prognosis group, the median cerebral artery pulsatility index of the good prognosis group decreased significantly, blood flow increased, and were statistically significant $(\mathrm{p}<0.05)$ (Table 4).

Draw the ROC curve of cerebral blood flow after CPR to predict the prognosis of cerebral function. The results showed that the area under the curve and the optimal critical value of cerebral blood flow were 0.731 and 5.69 . The sensitivity and specificity were $67.3 \%$ and $79.1 \%$ respectively (Figure 1 ). It indicated that cerebral blood flow is related to the state of brain function, which can predict the prognosis after CPR.

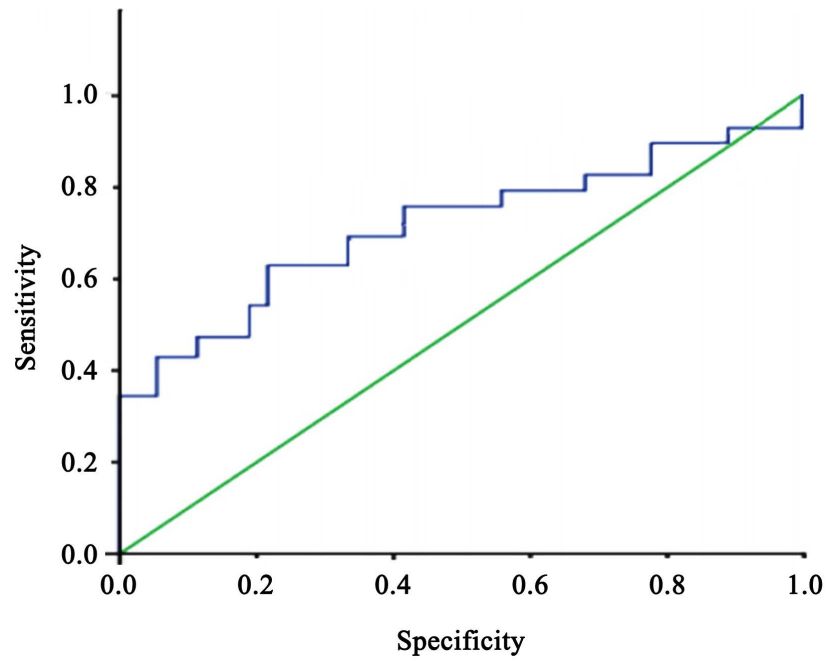

Figure 1. ROC curve of cerebral blood flow on prognosis evaluation of cerebral functional resuscitation after CPR.

Table 3. Comparison of cerebral blood flow parameters between the good prognosis group and bad group within $24 \mathrm{~h}(\bar{x} \pm \mathrm{s})$.

\begin{tabular}{cccc}
\hline Group $(\mathrm{n}=52)$ & $\mathrm{Vd}(\mathrm{cm} / \mathrm{s})$ & $\mathrm{Vs}(\mathrm{cm} / \mathrm{s})$ & $\mathrm{Vm}(\mathrm{cm} / \mathrm{s})$ \\
\hline Adverse group $(\mathrm{n}=21)$ & $26.1 \pm 15.4$ & $74.8 \pm 27.8$ & $42.9 \pm 18.1$ \\
the good prognosis group $(\mathrm{n}=31)$ & $43.5 \pm 30.1^{\star}$ & $112.1 \pm 44.3^{\star}$ & $70.1 \pm 34.0^{*}$ \\
t value & 3.520 & 3.657 & 3.842 \\
P value & 0.002 & 0.000 & 0.000 \\
\hline
\end{tabular}

*indicated significant difference compared with the poor prognosis group at $\mathrm{P}<0.05$.

Table 4. Central cerebral artery pulsatility index $(\bar{x} \pm s)$ and cerebral blood flow in 2 groups after CPR.

\begin{tabular}{ccc}
\hline Group & $\begin{array}{c}\text { PI of pulsatility index in } \\
\text { middle cerebral artery }\end{array}$ & Cerebral blood flow \\
\hline the good prognosis group $(\mathrm{n}=31)$ & $1.35 \pm 0.86$ & $5.14 \pm 1.13$ \\
Adverse group $(\mathrm{n}=21)$ & $1.95 \pm 0.66$ & $4.17 \pm 1.21$ \\
$\mathrm{t}$ value & -3.81 & 4.12 \\
P value & $<0.05$ & $<0.05$ \\
\hline
\end{tabular}




\section{Discussion}

Cardiac arrest is a common clinical emergency, which occurs in patients with cardiovascular disease, acute poisoning, cerebrovascular disease and respiratory diseases. Although more than half of the patients received standardized CPR and spontaneous circulation recovery was successfully restored [7]. But because the brain is in ischemia and hypoxia for a long time, the brain function may be completely lost, and the patient is still in an unconscious state. If the patient fails to treat in time, it may further lead to brain death and even seriously threaten to the patient's life and safety [8]. Therefore, the ultimate goal of CPR is to help patients with brain resuscitation, And how to get more targeted brain resuscitation, what to observe about the effect of brain resuscitation and how to judge the prognosis of patients objectively, has been the focus and difficulty for us [9].

Transcranial color bifunctional ultrasound (TCCD) can intuitively understand cerebral perfusion in patients after CPR by Dynamic monitoring of cerebral blood flow after CPR, such as diastolic blood flow velocity (Vd), systolic peak blood flow velocity (Vs), mean peak blood flow velocity (Vm), resistance index (RI) and pulsatility index (pulsatility index) of the middle cerebral artery [2] [3]. It helps to understand the extent of cerebral perfusion damage and to guide the clinical use of targeted treatment and observe the therapeutic effect, to assess prognosis. It also has many advantages, such as safety, non-invasive, fast, and repeated at the bedside, which are generally recognized. Compared with electroencephalography monitoring, TCCD is not susceptible to external causes $[10]$.

According to this study, in the good prognosis group, the patients whose consciousness were restored after CPR, the diastolic blood flow velocity $(\mathrm{Vd})$, systolic peak blood flow velocity (Vs) and mean peak blood flow velocity (Vm) were significantly increased in $24 \mathrm{~h}$ compared with the poor prognosis group ( $\mathrm{p}$ $<0.05$ ), the pulsatility index of middle cerebral artery decreased significantly in the good prognosis group ( $\mathrm{p}<0.05$ ), the value of which is used to predict the resistance; Draw the ROC curve of cerebral blood flow after CPR in predicting the prognosis of brain function. The results showed that the area and the optimal critical value of cerebral blood flow curve were 0.735 and 5.671, Sensitivity and specificity were $67.2 \%$ and $77.9 \%$, respectively, and this was consistent with most clinical reports [2] [3] [8] [10]. And it indicated that the prognosis of brain function after CPR can be predicted by cerebral blood flow.

Both cerebral hypoperfusion and cerebral congestion after CPR can cause secondary brain injury, which cannot maintain effective brain metabolism, while cerebral perfusion is closely related to intracranial pressure and cerebral blood flow. Therefore, in recent years, the evaluation of cerebral perfusion and brain function by dynamic monitoring of cerebral blood flow is advocated [10]. Other studies had found that the greater the blood flow measured by early transcranial Doppler ultrasound (TCCD), the better the prognosis of brain function in patients after CPR. It was proportional relationship between them. Detection of 
intracranial blood flow by TCCD can effectively prevent and reduce the repeated damage to the brain [10]. Although some reports showed that there were no significant differences between TCCD and other different ways for neurological prognostic groups and their predictive value needs further study [11]. However, from our study, it was still of practical clinical significance to dynamically monitor the changes of cerebral blood flow in patients after CPR, which had higher sensitivity and specificity.

\section{Conclusion}

The cerebral blood flow increase in the early stage of successful CPR is positively correlated with the prognosis of cerebral functional resuscitation. Monitoring intracranial blood flow after CPR has clinical value to evaluate prognosis of brain function by TCCD which is safe, non-invasive and repeated at the bedside.

\section{Limitations}

Although the results show a good predictive effect, due to the small sample size and the lack of prospective comparison with other monitoring methods, the conclusion has certain limitations. This is one of our next research directions.

\section{Conflicts of Interest}

All authors report no conflicts of interest related to this study.

\section{Author's Contribution}

Hui Guo wrote the paper; Jianguo Li carried out the conception of the article; Qian Zhao and Zhangshun Shen consulted the materials and revised them.

\section{Funding}

This study is supported by the 2019 Medical Science Research Project of Hebei Province (No. 20190202).

\section{References}

[1] Lee, B.K., Jeung, K.W., Song, K.H., et al. (2015) Prognostic Values of Gray Matter to White Matter Ratios on Early Brain Computed Tomography in Adult Comatose Patients after Out-of-Hospital Cardiac Arrest of Cardiac Etiology. Resuscitation, 96, 46-52. https://doi.org/10.1016/j.resuscitation.2015.07.027

[2] Wang, F. (2017) Research of Value by Transcranial Doppler Ultrasound in Detection of Cerebral Blood Flow in Cerebral Infarction Patients. Chinese Journal of Modern Drug Application, 11, 46-48.

[3] Xie, J. (2016) Assessment Value of Transcranial Doppler Hemodynamic Typing for Prognosis of Patients with Acute Middle Cerebral Artery Infarction. Journal of Hainan Medical University, 22, 1741-1744.

[4] Zhao, M., Duan, F., Lei, X., et al. (2016) To Explore the Diagnostic Value of Transcranial Doppler Ultrasound (TCD) in Patients with Early Cerebral Infarction. Chinese Journal of Practical Nervous Diseases, 19, 102-104. 
[5] Xue, J. and Geng, X.H. (2017) Diagnostic Value and Clinical Effect of Transcranial Doppler in Patients with Ischemic Stroke. Chinese Journal of Practical Nervous Diseases, 20, 50-52.

[6] Grossestreuer, A.V., Abella, B.S., Sheak, K.R., et al. (2016) Inter-Rater Reliability of Post-Arrest Cerebral Performance Category (CPC) Scores. Resuscitation, 109, 21-24. https://doi.org/10.1016/j.resuscitation.2016.09.006

[7] Frascone, R.J., Wayne, M.A., Swor, R.A., et al. (2013) Treatmalet of Non-Traumatic Out-of-Hospital Cardiac Arrest with Active Compression Decompression Cardiopulmonary Resuscitation plus an Impedance Threshold Device. Resuscitation, 84, 1214-1222. https://doi.org/10.1016/j.resuscitation.2013.05.002

[8] Xu, L.Y., Qiu, F., Shu, G.W., et al. (2017) Application of Transcranial Doppler Ultrasound in the Diagnosis of Early Cerebral Vasospasm in Patients with Craniocerebral Trauma. Chinese Journal of Integrative Medicine on Cardio/ Cerebrovascular Disease, 15, 2183-2184.

[9] He, X.C. and Yae, J.B. (2015) Study on Transcranial Doppler in Cerebral Vasospasm Caused by Subarachnoid Hemorrhage. Chinese Journal of Practical Nervous Diseases, No. 6, 1-3.

[10] Heimburger, D., Durand, M., Gaide-Chevronnay, L., et al. (2016) Quantitative Pupillometry and Transcranial Doppler Measuremalets in Patients Treated with Hypothermia after Cardiac Arrest. Resuscitation, 103, 88-93.

https://doi.org/10.1016/j.resuscitation.2016.02.026

[11] Expert Consensus Group on Early Neurological Function Prognosis Evaluation of Coma Patients after Cardiopulmonary Resuscitation. (2019) Expert Consensus on Early Neurological Function Prognosis Evaluation of Comatose Patients after Cardiopulmonary Resuscitation. Chinese Journal of Emergency Medicine, 28, 156-162. 